\title{
Coaxial Range Measurement - Current Trends for Mobile Robotic Applications
}

\author{
Martin D. Adams \\ School of Electrical \& Electronic Engineering \\ Nanyang Technological University \\ Singapore \\ email: eadams@ntu.edu.sg
}

August 28, 2001

\begin{abstract}
The useful environmental interaction of a mobile robot, is completely dependent on the reliable extraction of information from its immediate surroundings. A review of some of the most commonly used coaxial, active sensing methods in robotics is presented. A coaxial sensor results if the transmitter and receiver are geometrically arranged in a coaxial manner. Certain advantages of such configurations are discussed.

First, optical sensors, in which a controlled light signal is transmitted, are reviewed with respect to their applicability to mobile robotics research. A detailed overview of the literature describing light detection and ranging (LIDAR) systems for range measurement in robotics is given. In any LIDAR design, the physics of reflection of light from various surfaces must be addressed. Specular and/or diffuse reflection results which greatly affects a sensor's ability to measure range. The issues of the type of reflection, dynamic range of the received signal strength and crosstalk are addressed in relation to three popular LIDAR measurement techniques time of flight and frequency/amplitude modulated continuous wave methods.

A review of mobile robot research using sound navigation and ranging (SONAR) is presented where processing algorithms are demonstrated for the correct interpretation of ultrasonic data recorded in indoor environments. Once again, to understand the data provided by ultrasonic sensors, a model of the reflection mechanism is required. Ultrasonic wave reflection is generally dominated by specular reflections. Methods for the interpretation of SONAR data, for correct target classification, and hence range estimation, are given, based on the acoustic reflection mechanism. This requires SONAR scans of particular objects from multiple view points. Recent research has focussed on the use of arrays of SONARS to eliminate the necessity for multiple view point scanning, for the classification of targets. Methods will be presented which adopt multiple SONAR transducers to allow target identification and range estimation from a single view point.
\end{abstract}

Where possible, actual sensor data is presented to highlight the review.

keywords: Range sensing, LIDAR, SONAR.

\section{Introduction}

The foundation for any form of intelligent mobile robot navigation is based upon the perception of the environment by the robot. A sensor, or combination of sensors, accompanied by algorithms 
capable of automatically extracting useful information from it/them to make estimates about the current state of the robot's environment are required. Many mobile robot navigational algorithms are based upon the acquisition of robot to environmental object range information.

This article reviews two types of coaxial range measurement sensor, often used in mobile robotics research. During the past decade, a great deal of interest in light detection and ranging (LIDAR) systems is evident, and section 2 reviews the different methods of LIDAR sensing and presents results from working examples used in mobile robotics.

In section 2.1, the advantages of, and implementation issues involved in, coaxial sensor design are addressed in terms of correspondence and occlusion problems - terms which will be explained. Subsequently the physics of optical reflection is presented, along with a quantification of the large dynamic range of received signal intensities, which must be managed by these sensors (section 2.2).

Different LIDAR range estimation methods are explained in section 2.3. The effects of dynamic range and optical/electronic cross-talk on each technique, along with the advantages and disadvantages of the techniques themselves is discussed. Range scans taken from such devices are presented and analyses to give insight into the applicability of each ranging technique. References to working applications are also given with each technique.

Section 2.4 analyses the effect of averaging range data over time, for range estimate improvement. It is shown that a technique is available for improving range estimates in this manner, without loss of resolution in the resulting data. This method manipulates both the electronic constraints (in terms of the maximum sampling speed of a band-limited signal) and geometrical constraints (in terms of the optical foot print which is produced due to 'spreading' of the light beam) in any LIDAR.

No article on mobile robot sensing would be complete without an analysis of SONAR since, due to their low cost and ease of use, these sensors have been exploited in mobile robotics for more than 20 years.

Once again, section 3.1 begins by considering the physics of reflection of acoustic waveforms from various artifacts. Due to the specular nature of SONAR, models can be produced which allow the automatic interpretation of the data, from targets such as walls, corners, edges and cylinders, 
and subsequently the correct estimation of their range.

Initial work in robotics with SONAR has concentrated on the recognition of targets, which is necessary before range estimation can take place. This is based on the acquisition of data from multiple view points (section 3.2). Since this is rather a slow process, recent research has focussed on the use of SONAR arrays for the classification of targets without the necessity of moving the sensor. This is the subject of section 3.3.

Finally in section 3.4 some of the limitations in using SONAR, in terms of its beam-width and allowable data acquisition rates are explained.

Where possible, real sensor data has been used throughout the article to aid the understanding of the various methods covered.

\section{Light Detection and Ranging (LIDAR)}

LIDAR sensors are active devices which eliminate the correspondence ${ }^{1}$ problem associated with range estimation from stereo vision, and can also eliminate the disparity ${ }^{2}$ associated with stereo vision and active triangulation systems. The latter can lead to occlusion of an illuminated object from the receiver. Disparity can be eliminated in LIDAR sensors by constructing the device so that the transmitted and received light beams are coaxial. In robotic applications, LIDAR sensors usually consist of a transmitter which illuminates a target with a collimated beam, and a receiver capable of detecting the component of light which is reflected essentially coaxially with the transmitted beam. Often referred to as optical radars or LADARs (LASER detection and ranging), these devices produce a range estimate from the time needed for the light to reach the target and return. A mechanism sweeps the light beam to cover the required scene.

\subsection{Coaxial LIDAR Operation}

Light (usually near infra-red from an L.E.D. or laser) is collimated and transmitted from the transmitter $\mathrm{T}$ in figure 1 and hits a point $\mathrm{P}$ in the environment. For surfaces having a roughness

\footnotetext{
${ }^{1}$ The correspondence problem in photogrammetry can be defined as the problem of determining the pixels in two or more images, which correspond to a particular point in the environment - a problem which must be solved for range estimation.

${ }^{2}$ Disparity results from the offset between two or more cameras (or a transmitter and receiver) which can produce the problem where an object can be imaged in one camera (or illuminated by the transmitter) but not in the other (or is not visible to the receiver).
} 


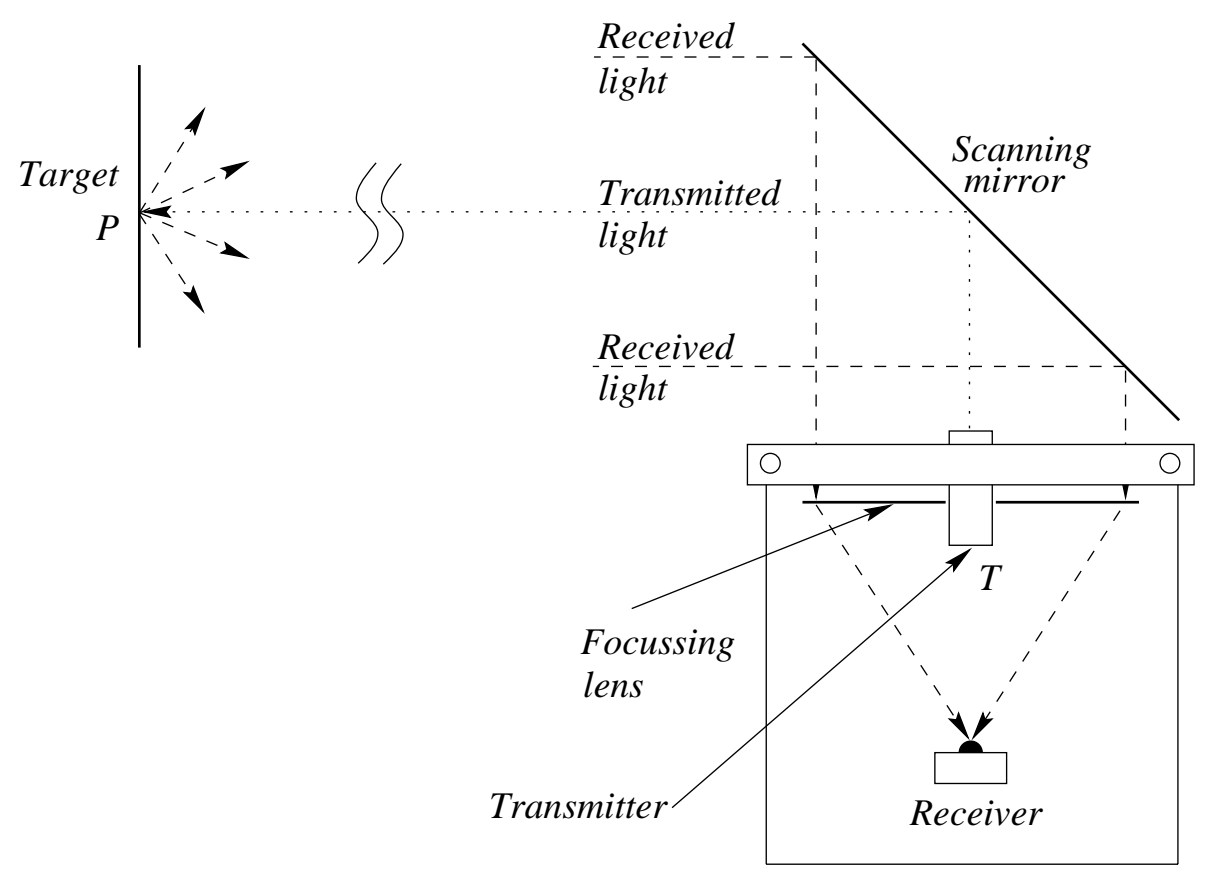

Figure 1: Coaxial light transmission and reception.

greater than the wavelength of the incident light, diffuse reflection will occur, meaning that the light is reflected almost isotropically. The physics of this reflection is fundamental to LIDAR design, and will be addressed in section 2.2. The wavelength of the light emitted is often in the range 800 to $1000 \mathrm{~nm}$ meaning that most surfaces with the exception of only highly polished reflecting objects, will be diffuse reflectors. This is because, if the wavelength $\lambda$ of the emitted wave is much less than the roughness $R$ of the surface, then primarily diffuse reflection occurs and is governed by Lambert's cosine law. An example of this is visible light (which has a spectrum of approximately $310 \mathrm{~nm}<\lambda<780 \mathrm{~nm}$ ) incident upon a wall. Since, in this case, $\lambda<<R$, the surface roughness of most walls, diffuse reflection occurs. Alternatively, if $\lambda>>R$ specular reflection occurs, governed by Snell's law. An obvious example of this is visible light incident upon an extremely flat surface — i.e. a mirror ${ }^{3}$. This phenomena will be referred to again in section 3 when SONAR sensors are discussed. In figure 1, the component of the infra-red light which falls within the receiving aperture of the sensor will return almost parallel to the transmitted beam, for distant objects.

\footnotetext{
${ }^{3}$ In real situations, both types of surface occur in which case reliable and untrustworthy range readings result. This is due to the unknown roughness of the surface encountered. This will affect the received signal amplitude which in turn provides a measure of the range estimate reliability. This means that, for subsequent processing of the range data, the surface properties are in fact irrelevant, and the signal amplitude alone quantifies the reliability of each range value.
} 


\subsection{Lambertian Reflection and Signal Reception}

When considering reflection from distant targets, Nitzan et al. presented a calculation of the received power expected at the receiver, based on Lambert's cosine law [1]. When incident upon an opaque surface, a light ray can under go specular reflection according to Fresnel's laws, and/or diffuse reflection governed by Lambert's cosine law. In practice, both occur simultaneously and it is the diffuse component which dominates the range estimate for most indoor surfaces, and which is of interest in LIDAR design.

If the transmitter produces an RMS radiant power $P_{T}$ which is incident upon a surface at an angle $\theta$ relative to the local surface normal (figure 2), the reflected power per steradian as a function of the angle $\theta$ is:

$$
I_{R}=\frac{P_{T} \rho \cos \theta}{\pi}
$$

where $\rho$ is the diffuse reflectivity of the surface, which is, in general, a function of the transmission wavelength.

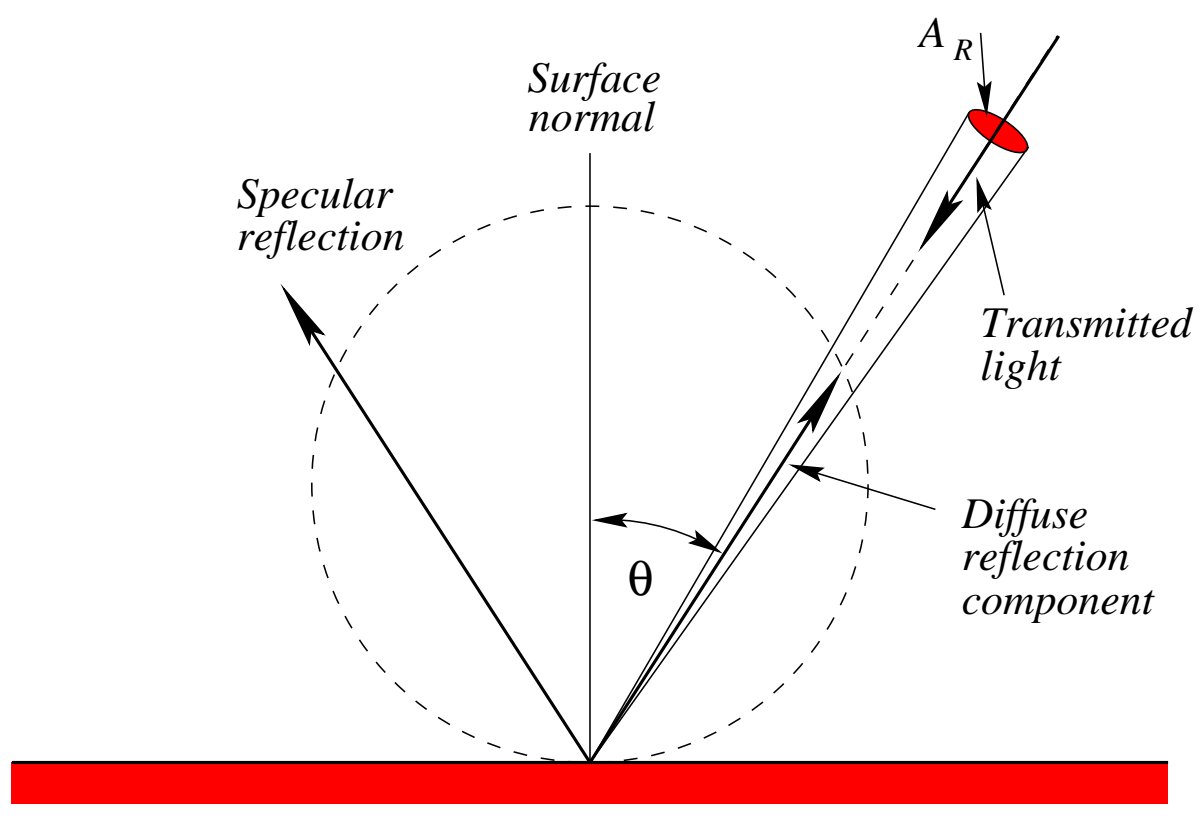

Figure 2: The variables which affect diffuse reflection according to Lambert's cosine law.

If the receiver aperture has an area $A_{R}$ and is situated a distance $r$ from the illuminated spot (figure 2), then it subtends a solid angle $\alpha$ given by:

$$
\alpha=\frac{A_{R}}{r^{2}}
$$


The total power then received is $P_{R}$ where:

$$
P_{R}=\eta \frac{A_{R} P_{T} \rho \cos \theta}{\pi r^{2}}
$$

where $\eta$ is the receiver's quantum efficiency.

It can be seen from equation 3 that the received power is proportional to $\rho \cos \theta / r^{2}$. Diffuse reflectance ratios $\rho$ can vary between approximately 0.02 for dark objects and almost 1.0 for white surfaces [1]. Further, if, for example in an indoor mobile robotic example, objects are to be visible to the sensor at incidence angles $0^{\circ}<\theta<80^{\circ}$ (i.e. near tangential reflection) and for ranges $0.2<r<15.0 \mathrm{~m}(0.2 \mathrm{~m}$ being being a typical path distance between the actual receiver and an object touching the sensor housing), the received signal can have a dynamic range of $1.620 \times 10^{6}: 1$ or $124 \mathrm{~dB}$. Nitzan states in his work that this dynamic range is in practice even higher, since at near normal angles of incidence, the specular component of the reflection can be detected, since this component is then reflected in a near coaxial manner with respect to the transmitted light beam [1]. Brownlow states that at close ranges, the effective field of view of the receiver must be taken into account and that the received power, predicted by Lambert's cosine law, is greatly over estimated, due to the inverse square relationship with the range $r$ [2]. In reality therefore, considering diffuse reflections only, the dynamic range will be less than the above estimate.

Equation 3 is fundamental to the design of a LIDAR. It places in question the correct starting point for the design. For example, if the aim is to operate an avalanche photo-diode (APD) based receiver at a low d.c. bias voltage (which improves its stability with respect to temperature), its responsivity will be reduced, meaning that a higher value for $P_{R}$ will be necessary to produce a detectable photo-current. This implies that the area of the photo-receiver, $A_{R}$ and/or the transmitter power, $P_{T}$ should be increased, yielding a larger and/or more expensive sensor.

\subsection{LIDAR Range Measurement Methods}

Three basic measurement categories dominate the market of commercially available LIDARs. The first, direct time-of-flight (TOF) measurement, measures the elapsed time for a light pulse to leave the transmitter and reach the receiver directly, first versions of which appeared as early as 1983 [3]. A second method is the frequency modulated continuous wave (F.M.C.W.) approach which measures 
the beat frequency between an FMCW signal and its reflection. For close range applications, and particularly in mobile robotic applications, a simple means of determining range is by measuring the phase shift between an amplitude modulated continuous wave (AMCW) and its received reflection. Variants based upon the A.M.C.W. method which use multiple transmitters to directly determine the orientation of objects, also exist and are explained in [4]. Other diverse variations of the above methods and some novel techniques are covered in $[5,6,7,8]$.

\subsubsection{Time-of-flight (TOF) Pulse LIDARs}

T.O.F. LIDARs transmit a pulse of light and directly measure the time taken for the echoed pulse to be received. The advantage of electro-magnetic pulse detection systems over their acoustic counterparts is that the speed of light is practically independent of humidity and temperature, meaning that no error compensation is required for the range calculation. In LIDAR systems, error due to temperature variations and electronic cross-talk are more of a problem. In particular the receiver, often an APD or photo-multiplier tube (PMT), is very dependent on the operating temperature. Electronics are required to compensate for this. A further issue is cross-talk between a transmitter and receiver, which can result from two effects:

1. Optical leakage - in which some of the transmitted light signal is internally reflected and reaches the receiver.

2. Electronic leakage - in which, due to the relatively high frequencies of the signals, part of the transmitted signal is electrically induced in the receiver.

Both of these leakage effects produce a "ghost" signal which will corrupt range estimates particularly from weakly reflecting targets [9]. Methods for electronic and optical shielding in LIDAR design are given in [10].

Various applications which make use of one dimensional direct TOF LIDARs exist. A system from Schwartz Electro-Optics called Auto Sense II detects moving cars from a fixed point and calculates their speed using a $50 \mathrm{~W}$ pulsed laser diode, and an APD for high sensitivity [11]. The same company has also produced a system named shield (scanning helicopter interference envelope 
laser detector), which serves the purpose of detecting objects within a $60 \mathrm{~m}$ radius, hemispherical region below the helicopter [12].

Indeed applications varying from 'tree sensing', for the detection and automatic pesticide spraying of trees, to the detection of foreign objects within a manipulators space for safety purposes, are abundant.

TOF LIDAR systems are relatively expensive due to the high speed and precision of the electronics necessary for timing the pulse transmission-reception time. For mobile robotic range sensing applications, where a resolution of a centimeter may be required, electronics capable of resolving picoseconds is necessary.

Potential sources of error in TOF systems include:

1. Variations in the speed of propagation - although in electro magnetic systems this can be ignored, this is not the case for acoustic systems (section 3).

2. Uncertainties in determining the exact time of arrival of the reflected pulse. As noted in section 2.2, the intensity of the received light pulse has a very large dynamic range which is dependent upon the sensor to target distance and the surface reflectivity. This can result in a returned waveform that sometimes has very little resemblance to the transmitted pulse.

3. Inaccuracies in the timing circuitry used to measure the round-trip pulse time.

4. Interaction between the incident wave and the target surface.

Over the past decade, SICK scanning TOF LADARS have been used in numerous vehicle research projects, ranging from underground mining [13] to road surface and kerb detection [14]. are documented. To shown some of the issues involved in the realistic detection of a light pulse, figure 3 demonstrates the form of the received pulse from a close target (at about $3 \mathrm{~m}$ distance) and a more distant target, in an outdoor environment. The instant in time when the pulse is considered detected, can be seen to be ambiguous in figure 3. Various electronic methods can be used to reduce this ambiguous effect. Vuylsteke has demonstrated that the detected waveform has a finite rise time which depends upon the received signal intensity, which somewhat complicates 


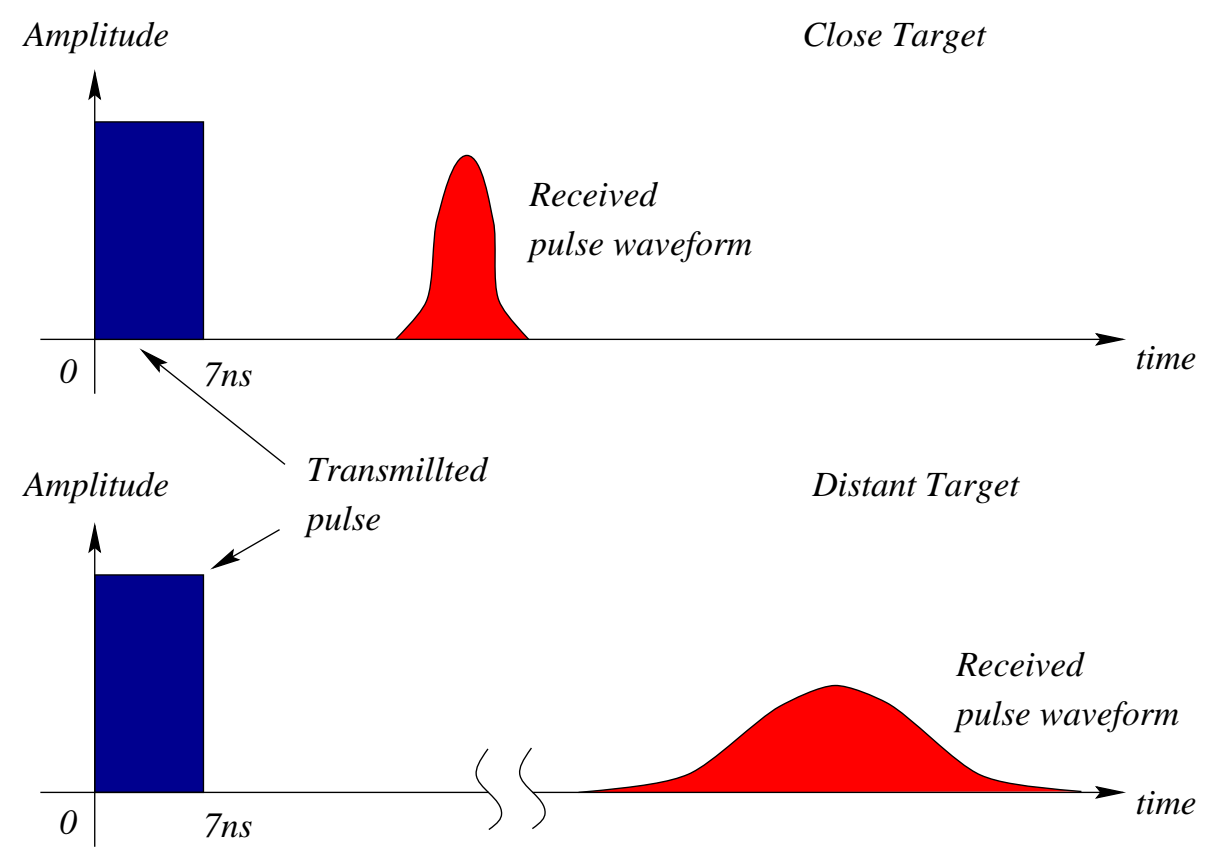

Figure 3: The form of the received pulses from near and distant targets from TOF LIDARS.

the electronic construction of the receiver since a constant fraction timing discriminator is required [15]. This determines the detector threshold to be some specified fraction of the peak value of the received pulse. This method functions well with particular rise time characteristics of the received pulse, meaning that not all returned waveforms, from any combination of target reflectance ratios, range and LIDAR transmitter to target angle of incidence, can be interpreted correctly, in terms of their exact time of reception. This is demonstrated in figures 4 to 6 . Figure 4 shows an image of a road surface in front of a slowly moving vehicle. The black crosses, across the surface, are recorded

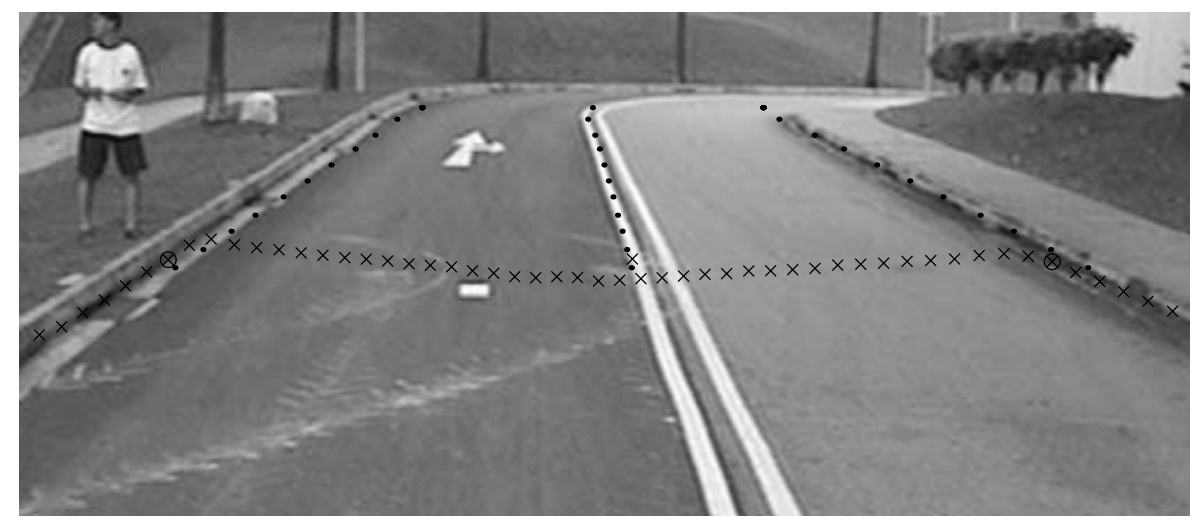

Figure 4: Road surface image and corresponding range points (crosses) recorded from a SICK TOF $L A D A R$. 
range values from a SICK TOF LADAR, which points downwards from the vehicle, so that the light beam intersects the surface at about $10 \mathrm{~m}$.

Figure 5 shows a plan view of the recorded range readings. Note that the curve in the range

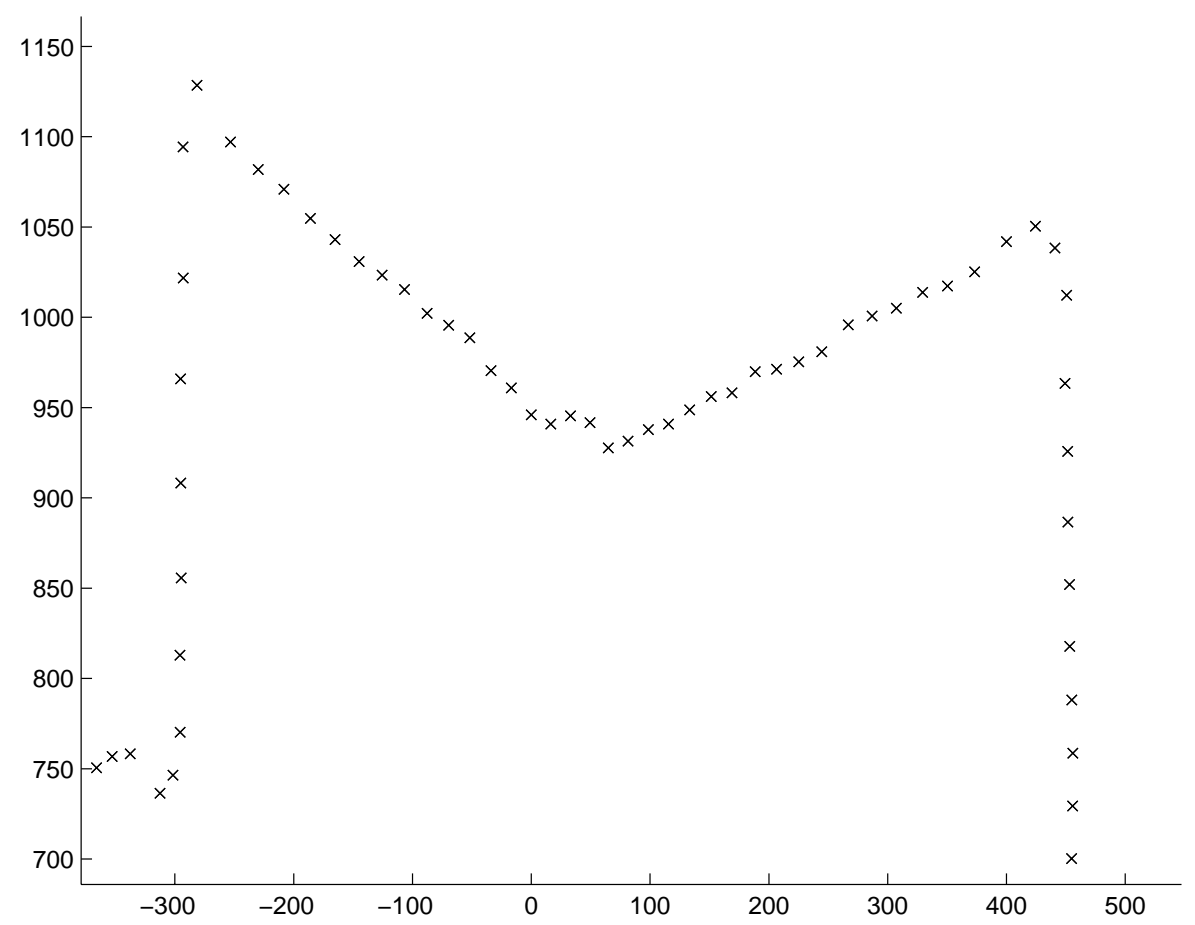

Figure 5: Plan view of the range readings recorded from the road surface. The vertical axis points along the direction of the road and the horizontal axis points across the road. Both scales represent distance in $\mathrm{cm}$.

values results from a quite extreme curvature in the road surface.

Figure 6 shows the results of the same experiment, conducted with the sensor tilted more downwards, so that the range intersection with the road surface is only at a bout $3 \mathrm{~m}$. Firstly it can be seen that the curvature appears less pronounced - this is to be expected, due to the geometrical aspect ratio of the intersection of the scanned LASER with the curved road surface. Secondly however, the range values along the surface, appear in groups of severely distorted values. In this case the received signal pulse amplitude is high (close range, near normal angle of incidence between road surface and LASER) and the sensor pulse detection electronics have been tuned to correctly interpret weaker received pulse waveforms (which result from the more distant range values in figure 5) rather than larger signals. This results in the obscure range values shown in figure 6 as the time of detection of the pulse becomes distorted. Hence, even in TOF LIDARS, it is necessary to specify 


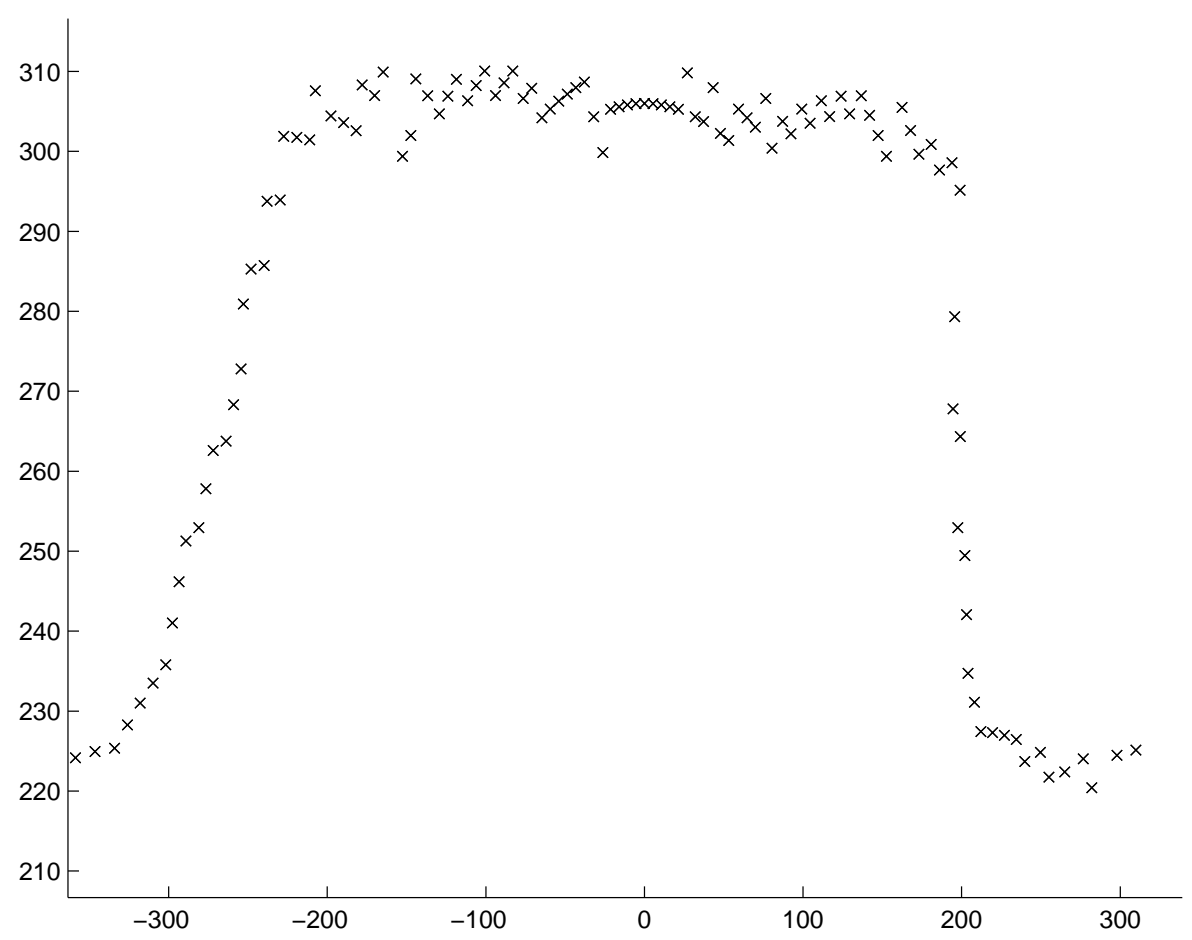

Figure 6: Plan view of range readings recorded from the road surface, with the LADAR's LASER intersecting the surface at about $3 \mathrm{~m}$. The vertical axis points along the direction of the road and the horizontal axis points across the road. Both scales represent distance in $\mathrm{cm}$.

the dynamic range of received signal intensity over which the LADAR can reliably operate.

\subsubsection{F.M.C.W. LIDARs}

The necessity for high speed electronics can be removed by transmitting a continuous wave of light energy. To measure range, it is possible to frequency modulate the light produced by the transmitter meaning that a range of frequencies is transmitted which varies linearly with time. This principle is shown in figure 7 , where the frequency as a function of time is:

$$
f(t)=f_{0}+a t
$$

where $a$ is a constant and $t$ is the elapsed time. The received signal is received a time $T$ after transmission and has a frequency variation with time which is shifted a time $T$ along the time axis of figure 7 where $T$ is given by:

$$
T=\frac{2 d}{c}
$$

where $d$ is the distance to target and $c$ is the speed of light. 


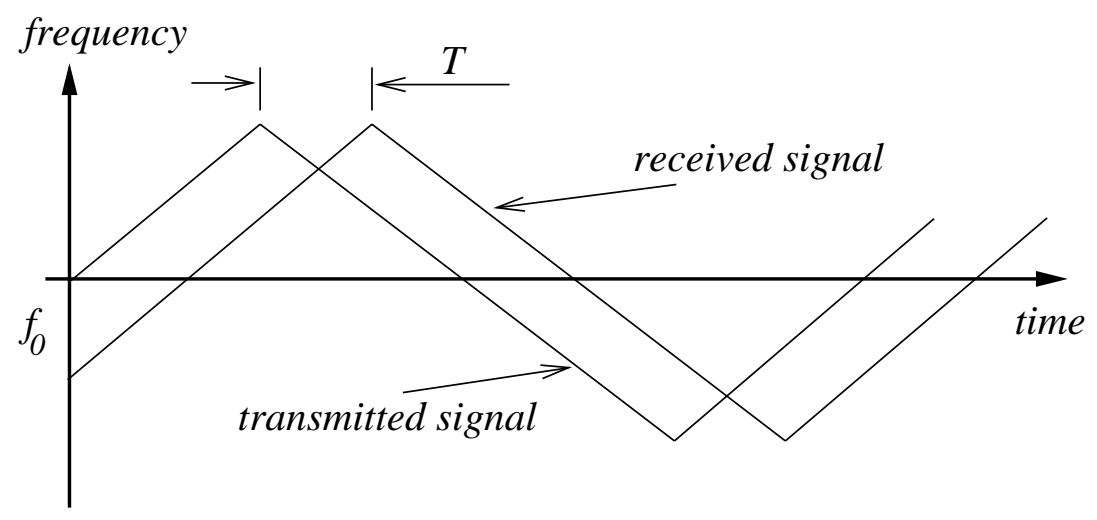

Figure 7: F.M.C.W. range estimation.

By mixing and filtering the transmitted and received signals, the beat or difference frequency results which is directly proportional to range. Distance measurement is therefore as accurate as the linearity of the frequency variation over the counting interval. This type of modulation, modulates the frequency of a constant amplitude sinusoidal intensity variation. It is also possible however to frequency modulate the natural sinusoidal variation in intensity produced by a LASER diode. Advances in wavelength control of LASER diodes now allow the wavelength to be shifted by varying its temperature, a technique used previously in RADAR techniques.

F.M.C.W. LIDARs have found an application as an aid to driving in the automotive industry. A company named Vorad Technologies has developed an F.M.C.W. system which can be mounted on the front of a vehicle and measure the range to, and speed of other traffic [16]. A low powered $(50 \mathrm{~mW})$, high frequency (center frequency $=24.725 \mathrm{GHz})$ modulated gun diode is used as the transmitter and the system is reported to be able to measure range up to about 100 meters.

An F.M.C.W. microwave sensor, again to aid driving in particular with respect to the drivers blind spot, has been developed by "Safety First Systems Ltd". This system adopts a modified F.M.C.W. technique, where the modulating frequency has $f_{0}=10.525 \mathrm{GHz}$, and a $50 \mathrm{MHz}$ bandwidth, to improve the resolution over conventional F.M.C.W. techniques [17].

Relatively few F.M.C.W. LIDAR sensors exist, since it is, in practice, difficult to achieve a linear frequency - time optical transmission signal, which operates reliably about the high transmission frequencies necessary. Therefore, although many radar systems adopt the F.M.C.W. principle, optical sensors are usually based on the T.O.F. or A.M.C.W. principles. 


\subsubsection{A.M.C.W. LIDARs}

For close range applications, a simple means of determining the time of flight of the light is by measuring the phase shift between an amplitude modulated continuous wave (A.M.C.W.) and its received reflection.

These sensors should theoretically transmit $100 \%$ amplitude modulated light at a known frequency and measure the phase shift between the transmitted and reflected signals. Figure 8 shows how this technique can be used to measure range. The wavelength of the modulating signal obeys

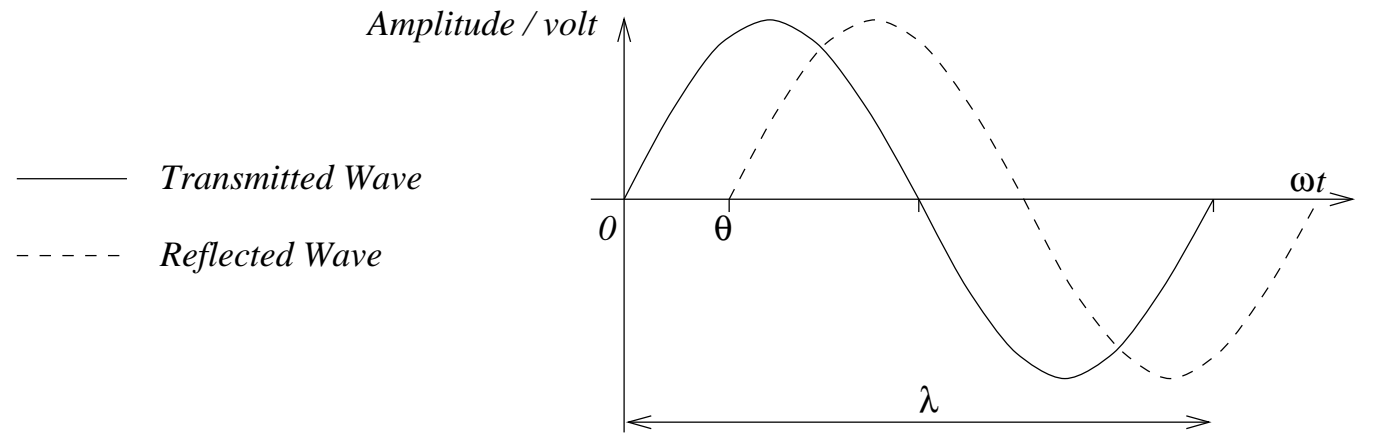

Figure 8: Range estimation by measuring the phase shift between transmitted and received signals.

the equation $c=f \lambda$ where $c$ is the speed of infra-red light and $f$ the modulating frequency. For example, in an indoor application, one could choose $f=10 \mathrm{MHz}$, giving $\lambda=30$ meters. The range from the sensor to the target is given by:

$$
\text { range }=\frac{\theta}{4 \pi} \lambda
$$

where $\theta$ is the electronically measured phase difference between the transmitted and reflected light beams, and $\lambda$ the known modulating wavelength. It can be seen that the transmission of a single frequency modulated wave can theoretically result in ambiguous range estimates since, for example if $\lambda=30$ meters, a target at a range of 5 meters would give an indistinguishable phase measurement from a target at 20 meters, since each phase angle would be $360^{\circ}$ apart ${ }^{4}$. An 'ambiguity interval' of $\lambda / 2$ therefore exists.

Figure 9 shows raw data recorded from an AMCW scanning LIDAR, where the left scan shows the amplitude of the received signal in polar coordinates (radial axis in volts) as a function of the scanning angle (angular coordinate in degrees), and the right scan shows the range output (plotted

\footnotetext{
${ }^{4}$ Note that the wave has to travel to the target and back meaning that the disambiguous measurable range is $\lambda / 2$
} 
in Cartesian form) [10]. The actual hand measured environment is shown as the dotted lines in the
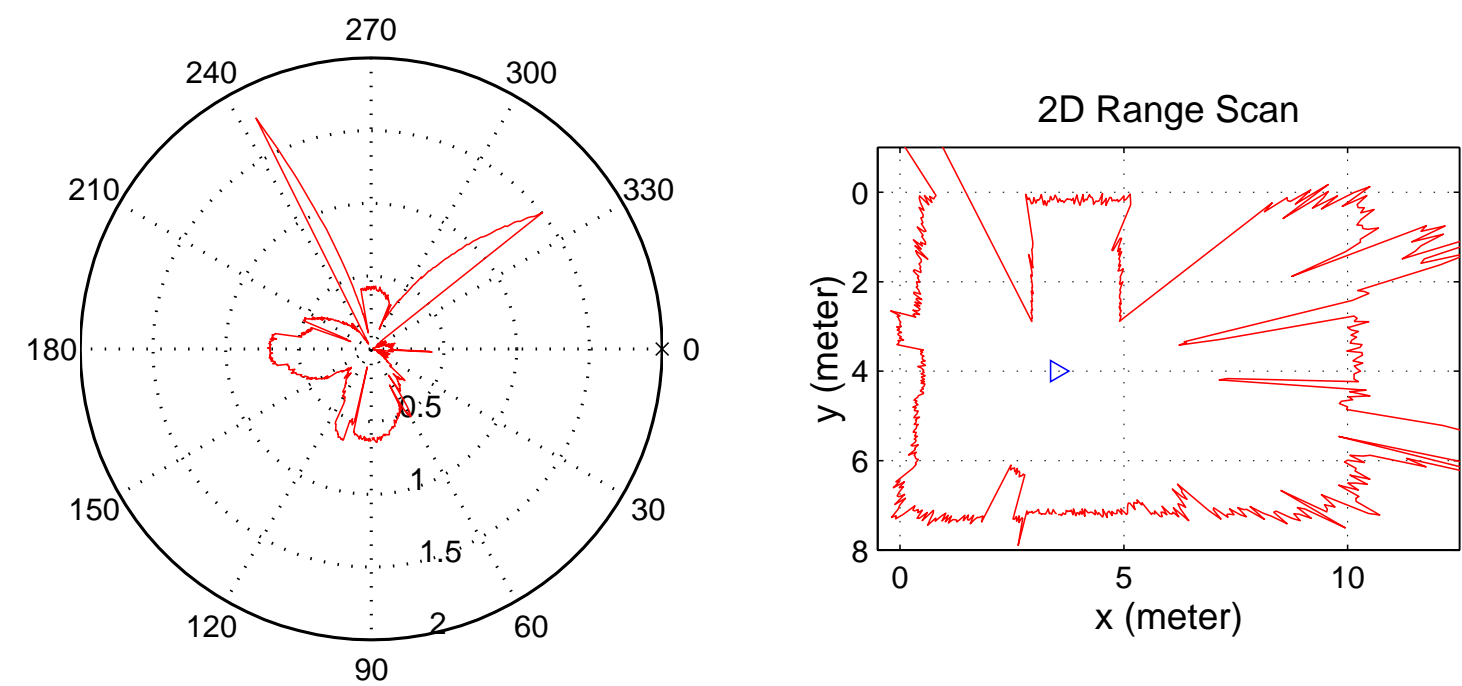

Figure 9: Left: Signal amplitude (radial axis (volts)) versus sensor scanning angle (degrees). Right: Range output shown as dots in Cartesian coordinates. The triangle in the right graph shows the position of the mobile robot.

right figure, and the actual range data as points. It can be seen that even the raw data provided by the LIDAR forms a good representation of its environment. The left figure shows the amplitude as a function of the scanning angle in polar coordinates, which corresponds to how much light was received at each bearing.

Miller and Wagner first suggested a link between the received signal amplitude and the range uncertainty in AMCW range detection [18]. A complete model, providing range variance and systematic error, as a function of received signal amplitude and various other sensor electronic noise sources, was provided by Adams in 1992 [19]. In theory, phase shift in an AMCW LIDAR varies with range only. However, in practise, the dynamic range of received signal intensity can introduce phase shifts within the receiver electronics. This can be accounted for and corrected with correct calibration procedures [20].

Results using the derived model mentioned above, are shown in figure 10 where the left scan shows the amplitude of the received signal as a function of the scanning angle (similar to the environment in figure 9) and the right scan shows the standard deviation in range (plotted in Cartesian form). The right scan shows lines of length $2 \sigma_{r}$, calculated from the range variance as a 

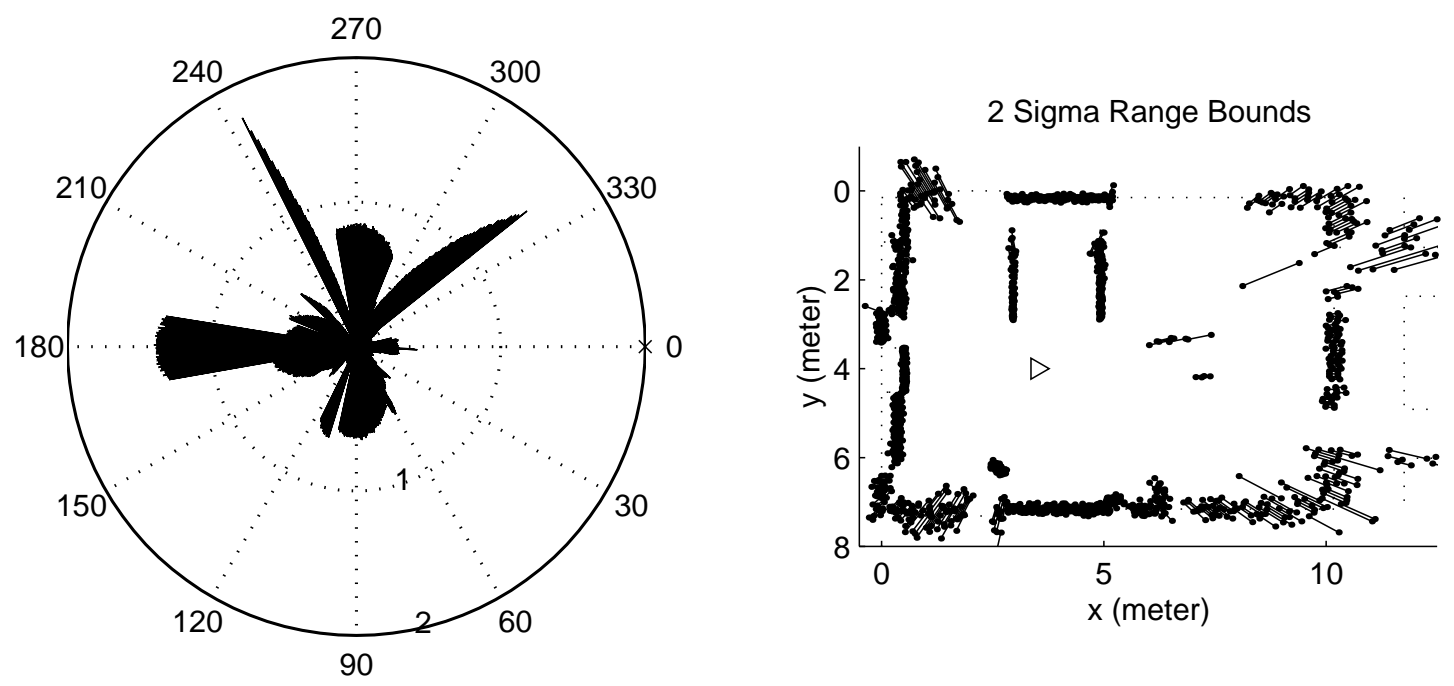

Figure 10: Left: Signal amplitude (radial axis (volts)) versus sensor scanning angle (degrees). Right: Lines of length $2 \sigma_{r}$ centered on the range estimates. The triangle in the right graph shows the position of the mobile robot. The dotted line represents a plan view of the actual environment.

function of received signal amplitude (left scan) derived from an AMCW LIDAR physical model, centered on the actual range estimates themselves [19]. Again the dotted line represents a plan view of the actual environment. The length of the line segments in figure 10 gives a quantitative assessment of the uncertainty associated with each range estimate, particularly useful for weighting the influence of each range value for feature detection purposes [10].

\subsection{LIDAR Data Improvement - Averaging Range Data}

Given enough time, several range signal samples can be averaged to form a single range estimate with lower variance but no loss of angular resolution in the scan $[2,19]$. In mobile robotic applications however, a LIDAR is usually used in continuous scanning mode, meaning that each range sample could result from a different environmental object. All LIDARs produce a finite optical footprint, rather than an infinitesimal point, on the object(s) being sensed. The sampled range data can therefore be considered to be the output of a dynamic system, as no matter what range changes actually occur, the range signal cannot change instantly because of two constraints:

1. Electronic Constraint: The output range signal is the result of low pass filtering the phase estimate and is therefore band-limited, thus limiting the speed at which new range information 
can be sampled.

2. Geometrical Constraint: Due to the finite size of the optical footprint, the range signal results from the convolution of all reflected light signals within the footprint, thus lowering the angular resolution [10].

The aim of this section is to manipulate these two effects to optimize the sampling and averaging of the range output. Constraint 1 will be quantified to give the number of consecutive samples to be averaged, resulting in a single point with lower range variance than the individual samples. Constraint 2 will then be quantified to ensure that no loss of angular resolution results in the new, lower density scan. This is important in applications such as feature extraction, where the location of edges needs to be known, with some precision, for mobile robot localization [10, 21].

Quantification of Constraint 1: It can be shown that if the sampling time interval $\Delta T$ is much less than the low pass filter time constant $T_{f}$ (high correlation between successive samples) then the standard deviation $\bar{\sigma}_{r}$ of the average of $n$ samples taken at time intervals $\Delta T$ is:

$$
\bar{\sigma}_{r}=\frac{\sigma_{r}}{\sqrt{n_{\mathrm{eff}}}}
$$

where $\sigma_{r}$ is the standard deviation of each individual sample. For a single pole, low pass filter, if $n \Delta T<<T_{f}$, then $n_{\mathrm{eff}} \approx 1[1]$. If however:

$$
n \Delta T>>T_{f}
$$

$n_{\text {eff }} \approx\left(n \Delta T / 2 T_{f}\right)$. Note that this result is only true for $\Delta T<<T_{f}$ and in any case, $n_{\text {eff }}$ can never be larger than $n$, the number of samples recorded. Hence if a target can be sampled such that the product $n \Delta T$ is greater than $2 T_{f}$ an improvement in the confidence in the range estimate results, since $\bar{\sigma}_{r}$ will be lower than $\sigma_{r}$.

The above criterion was used to reduce the range error in figure 11 where two 3D scans are shown. The left scan shows a corner of an environment containing cupboards and a chest of drawers, each sample taken every $0.5^{\circ}$ of sensor head rotation (every $0.7 \mathrm{~ms}^{5}$ ). Every 4 of these were averaged to form a single data point in the right range map. The improvement in the range

\footnotetext{
${ }^{5}$ since the sensor head was scanning at 2 revolutions per second about its vertical axis
} 

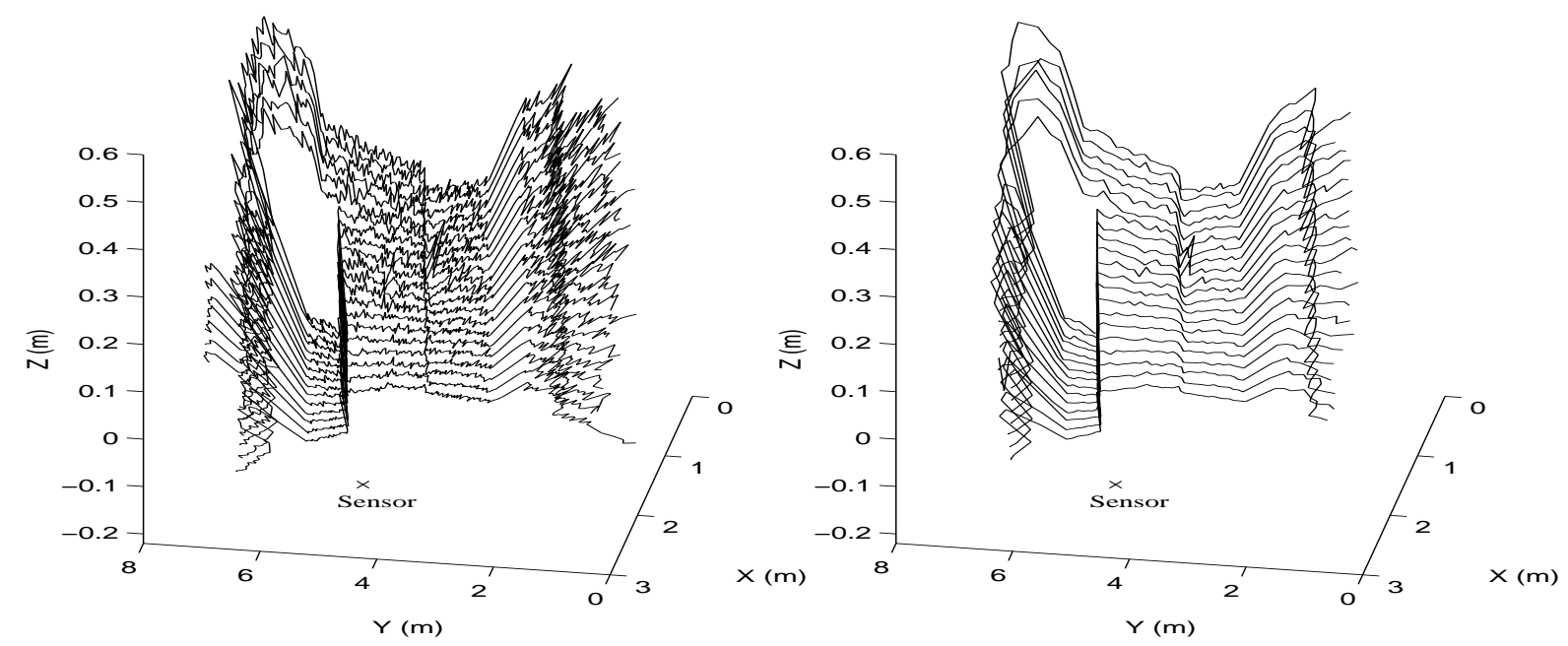

Figure 11: 3D range data showing the corner of a room from a single $3 D$ scan. The left scan shows all recorded data points from the section under consideration, and the right scan shows only one sample averaged from every 4 horizontally scanned range points.

variance is evident. In this case $4 \Delta T=2.8 \mathrm{~ms}$, which is about ten times larger than $T_{f}$, which in the design example is $0.28 \mathrm{~ms}$, corresponding to a filter cut off frequency of $3.5 \mathrm{kHz}$.

Quantification of Constraint 2: To produce these results with no loss of angular resolution, the time necessary to record one full $2 \mathrm{D}$ section of a scan, $T_{\text {scan }}$, has a lower limit. This is because (for no resolution loss) the averaging should be restricted to a scanned area smaller than, or equal to, the optical footprint, i.e. expressed as a time constraint: $n \Delta T \leq$ optical footprint traversal time, $T_{\text {footprint }}$. From geometrical considerations [10]:

$$
T_{\text {footprint }}=\frac{b T_{\text {scan }}}{\pi R \max }
$$

where $b=$ optical footprint radius at maximum range $R_{\max }$. Substituting for $n \Delta T$ in inequality 8 and resolving for the $2 \mathrm{D}$ scan time $T_{\text {scan }}$ gives:

$$
T_{\text {scan }}>>\frac{\pi R_{\max } T_{F}}{b}
$$

For the LIDAR used in figure $11, R_{\max }=15.0 \mathrm{~m}, T_{F}=0.28 \mathrm{~ms}, b=0.05 \mathrm{~m}$ (beam radius at $15 \mathrm{~m}$ range), meaning that the lower limit for $T_{\text {scan }}=0.26 \mathrm{~s}$. The $2 \mathrm{D}$ scanning rate of 2 revs $/ \mathrm{s}$ just satisfies this so that no loss in resolution is observed in the right scan of figure 11. 


\section{Ultrasonic Range Sensing}

The introduction of the Polaroid SONAR range sensor as a focusing aid for Polaroid cameras, sparked a trend within the mobile robotics community which made use of these devices for creating range maps in indoor environments, with articles, such as that from Koenigsburg, dating back as early as 1982 [22]. Notable methods for robot navigation using Polaroid and other SONAR devices are also given in $[23,24,13,25,26]$. Indeed their use has become so common, that an article on sensing methods in robotics would not be complete without a review of ultrasonic range sensors and the interpretation of their data.

The Polaroid device, and many of the other electro-static/piezo-ceramic SONARs, operate using the time of flight (TOF) principle $[27,28]$. In its most common form, it comes as a single transducer, which initially operates as a transmitter, transmitting an acoustic wave into the environment, and then switches its mode to that of a receiver in order to receive the acoustic wave after reflection from an object. A range reading results when the returned echo's amplitude exceeds a predefined value, this occurring a time $T$ after transmission. The estimated range $r$ is then simply:

$$
r=\frac{v T}{2}
$$

where $v$ is the speed of sound in air, assumed constant. By scanning the SONAR sensor about a vertical axis, a $2 \mathrm{D}$ polar coordinate range map results.

Figure 12 shows such a polar coordinated range scan taken in an indoor environment [29]. This data was recorded from a single electrostatic transducer SNT device which has a maximum range of $5 \mathrm{~m}$ [28]. The characteristics, with respect to beam width and frequency of the transmitted wave, are similar to those of the Polaroid SONAR, and many others used in mobile robotics. The dashed lines show a simple line model of the actual environment, and the solid line represents the actual range data recorded from the ultrasonic sensor, the sensor itself being positioned at the center of the cross $(+)$. At first sight, the range map seems to be a poor representation of its environment. It will however be demonstrated that once the data is correctly interpreted, certain useful information can be obtained from SONAR.

A paper fundamental to the understanding of SONAR range measurements in air, taken from 


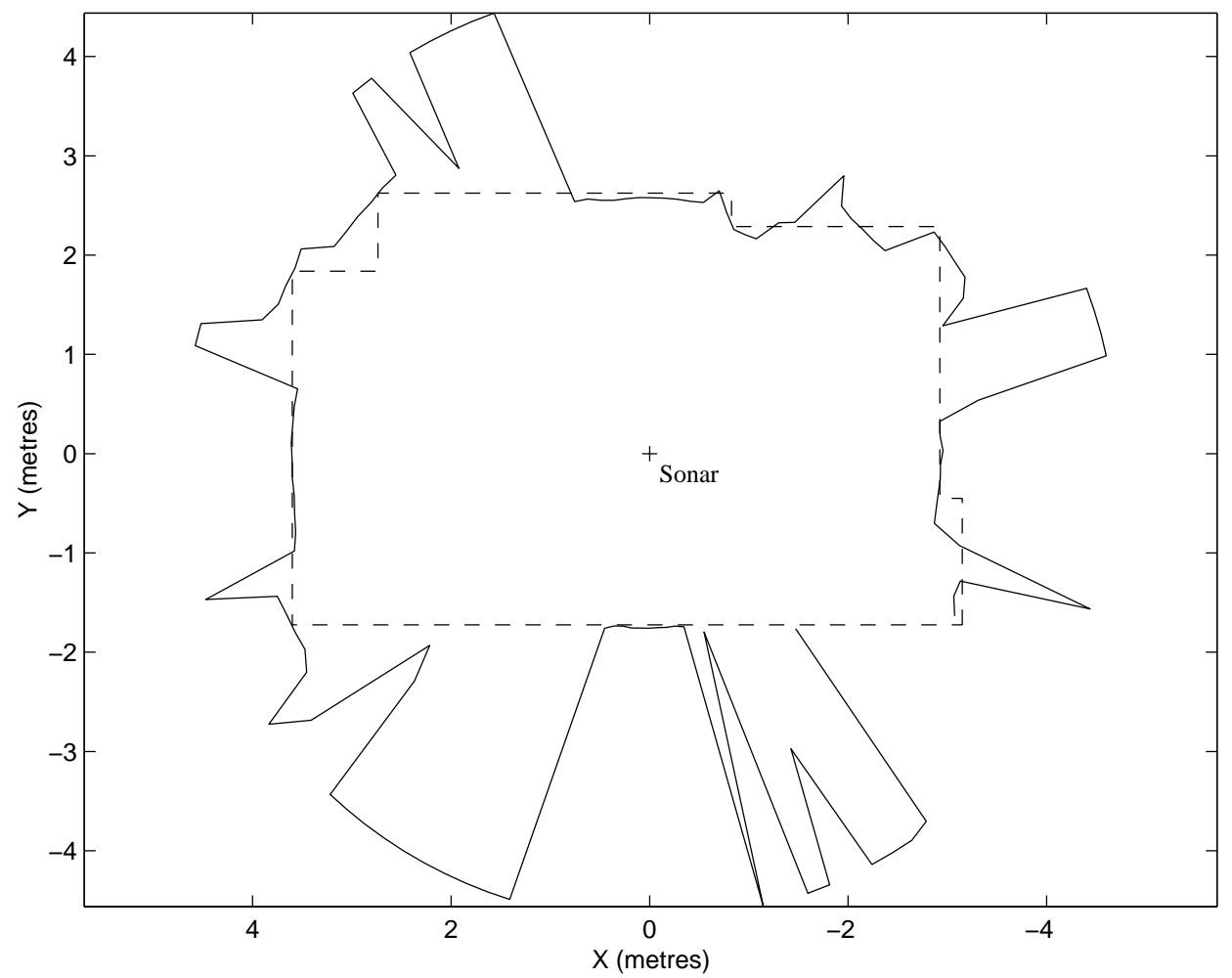

Figure 12: SONAR range data recorded in a simple laboratory environment. The dashed line corresponds to the actual hand measured environment and the solid line represents the SONAR range data, one point being recorded for every $2.8^{\circ}$ of rotation.

a single transmit/receive SONAR transducer, was presented by Kuc and Siegel as early as 1987 [30]. In this paper, a qualitative model of SONAR behavior was explained and some of the basic properties and the physics behind ultrasonic reflection from various indoor surfaces now follows.

\subsection{SONAR - The Physics of Reflection}

The first point to be noted with SONAR is that no time of flight range value can be produced if the detected signal amplitude does not exceed a preset threshold value ${ }^{6}$. With the Polaroid module, this threshold is automatically decreased as a function of the time after the sound wave is transmitted [27, 28]. This allows for the attenuation of ultrasonic radiation in air. Interesting work by Birsel and Barshan addresses the effect of noise upon an ultrasonic echo's amplitude using modeling methods based upon evidential reasoning [32].

Secondly, the longitudinal pressure wave emitted by an ultrasonic transmitter has a wavelength

\footnotetext{
${ }^{6}$ Barshan has examined alternative methods for received waveform detection based on curve fitting. This will be reviewed further in section 3.3 [31].
} 
of the order of several millimeters. In general, when any wave is incident upon a surface, two modes of reflection are possible, namely specular or diffuse, as discussed in the section on optical reflection (section 2.2. In general, both types of reflection occur simultaneously, but the tendency to favor one mode of reflection over the other is dependent upon the wavelength of the incident wave compared with the roughness of the surface.

Since SONAR has a wavelength $\lambda$ within the millimeter range (the Polaroid device produces an ultrasonic wavelength $\lambda \approx 7 \mathrm{~mm}$ ), for most indoor surfaces, $\lambda>>$ and indeed specular reflection occurs. Hence, to visualize how SONAR 'sees' its environment, it is easier to think of an optical line of sight range finder scanning within a hall of mirrors. Due to specular reflections, only readings which are recorded in a perpendicular sense to indoor surfaces will be correct. Large angles of incidence between the SONAR's center line and the surface normal produce over estimates in range. This is because the sound wave undergoes total internal reflection several times before eventually reaching the transducer. This explains the form of the SONAR scan data recorded in figure 12 .

Another effect to be noted with SONAR is its beam width. Unlike most optical sensors, the transmitter and receiver of an ultrasonic sensor are not usually focussed (an exception to this being the work of Crowley, in which a SONAR is focussed [33]). This means that the first signal above the threshold amplitude received anywhere within a certain conical region emerging from the transducer produces the range reading. For a given size of transducer, the beam width or, more precisely, the acoustic aperture of the sensor, increases in an inversely proportional sense with respect to the frequency of the emitted radiation. For example, one detailed model of the Polaroid SONAR package shows $-80 \mathrm{db}$ receiver attenuation at angles of $\pm 12^{\circ}$ at a frequency of 49.4 $\mathrm{kHz}$ (which corresponds to an acoustic wavelength of approximately $7 \mathrm{~mm}$ ) [34]. A consequence of this effect is demonstrated in figure 13. Although the transducer's center line is aimed at an angle $\psi$ to the perpendicular of the nearby wall, the measured distance will be $d_{\text {wall }}$ and $n o t d_{\text {true }}$ for angles $-12^{\circ}<\psi<12^{\circ}$. This is because the first part of the wave from the transmitted sound cone to return to the transducer travels along path $\mathrm{OA}$ and back to $\mathrm{O}$ and not along path $\mathrm{OBO}$. This explains the curved regions near the walls, visible in figure 12 . This is demonstrated with real 


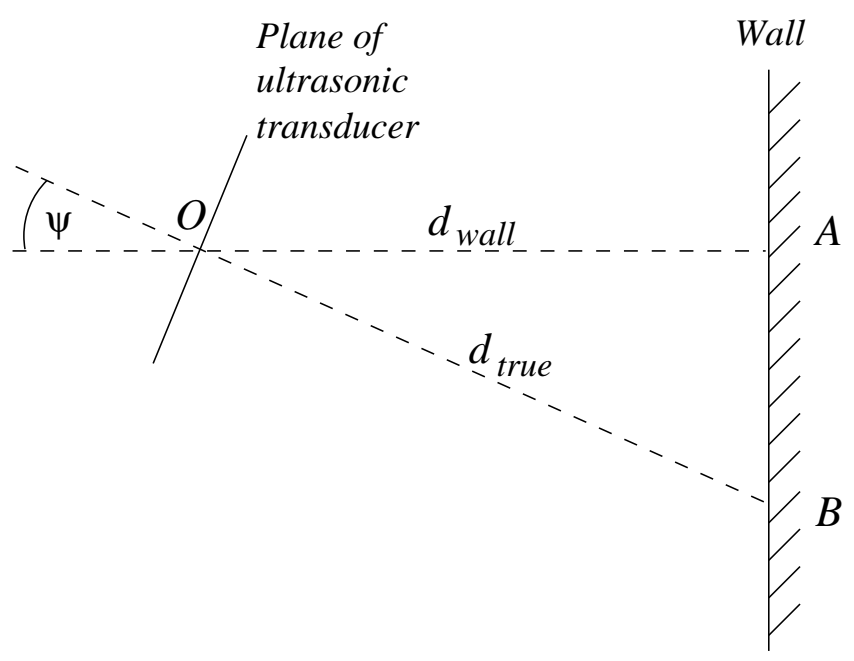

Figure 13: The geometry of ultrasonic reflection in the vicinity of a wall.

SONAR data in figure 14 where the walls as 'seen' by the SONAR are marked ' $W$ '. This effect was noted by Kuc and Siegel, where they state:

"For a wall to be visible, the transmitter/receiver location within the space must have an unobstructed perpendicular projection to that wall."

The curved regions produced in the vicinity of the walls (as can be seen in figure 14) were referred to as regions of constant depth (RCD's) in Leonard's work [26]. Indeed it was suggested by Kuc and Siegel and Leonard and Durrant-Whyte that the correct interpretation of SONAR data begins by extracting RCD's from SONAR scans. Kuc and Siegel went on to consider corners and $\operatorname{edges}^{7}$.

An interesting effect can be observed when scanning in the vicinity of a corner. This is demonstrated by the geometry in figure 15. It can be seen that any transmitted wavefront within the sound cone will produce a range reading equal to $d_{1}+d_{2}+d_{3}$. For a $90^{\circ}$ corner, simple geometry shows that $d_{1}+d_{2}+d_{3}=2 l$ for all transducer angles $\gamma, l$ being the actual perpendicular distance, with respect to the transducer, to the corner. Hence the form of the detected signal is the same as that from a wall, and the transducer inclination angle is again irrelevant. Hence the second part of the model presented by Kuc and Siegel states:

"For corners to be visible, the transducer must have an unobstructed line-of-sight to their location."

\footnotetext{
${ }^{7}$ a corner being the concave dihedral formed by the intersection of two perpendicular walls and an edge the resulting convex dihedral.
} 


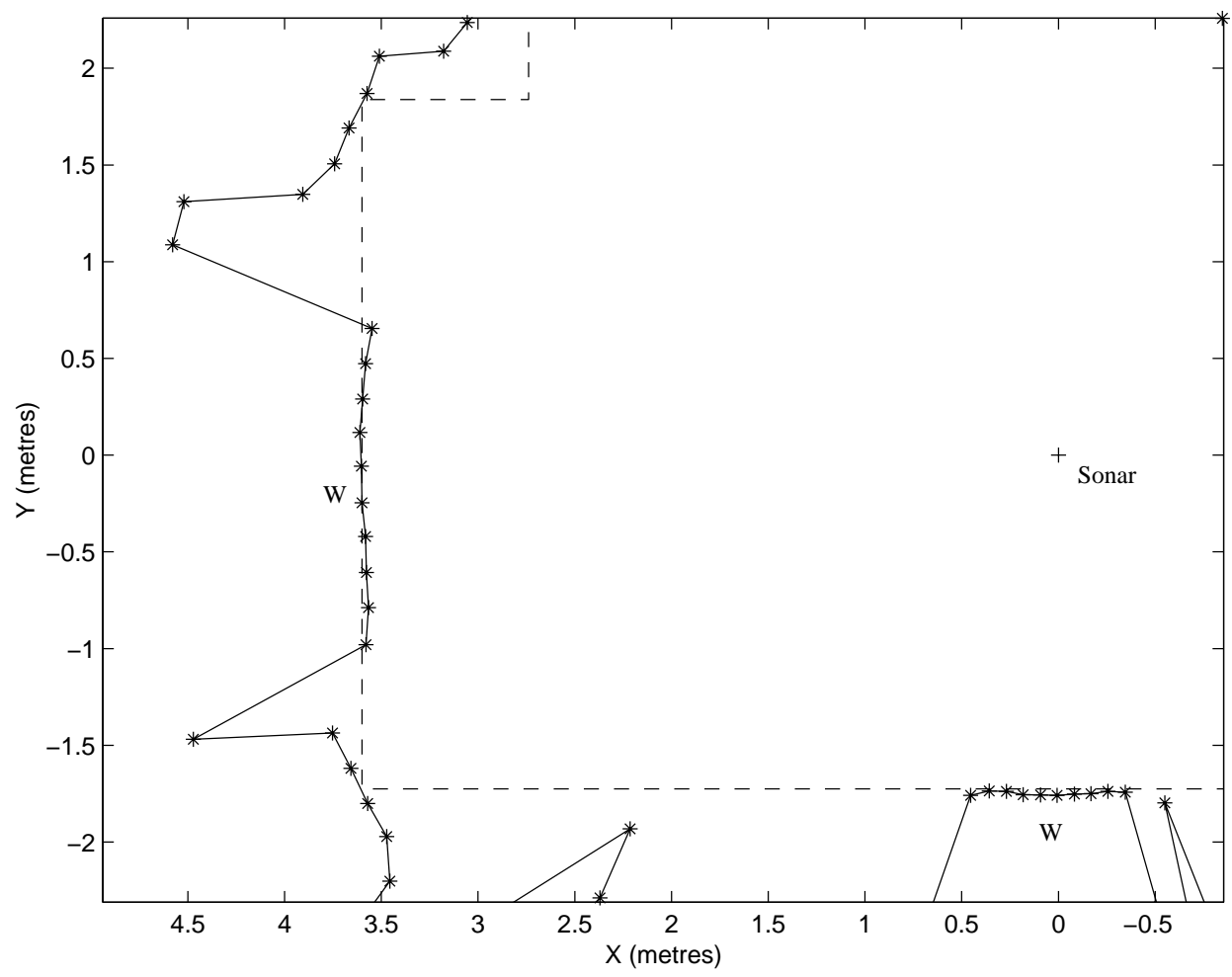

Figure 14: SONAR range data points recorded as the transducer is scanned passed two walls (marked ' $W$ ').

Indeed because of this property, even very small $90^{\circ}$ corners such as door frames, form very strong reflectors for SONAR and are termed retro-reflectors.

\subsection{Target Classification from Multiple View-Point Scans}

This model of ultrasonic reflection in air, has formed the basis for most of the subsequent research in the field. Continuing the trend set by Kuc and Siegel [30], it seems to be the norm that targets are statistically classified into four reflector types: planes, corners, edges and unknown. Leonard and Durrant-Whyte further characterized SONAR data recorded from cylinders [30, 26]. The aim of this analysis was to fuel some form of navigation/map building process. Clearly from the discussion presented so far, from a single transmit/receive transducer, it is impossible to determine whether the RCD's extracted from a single scan are produced by walls or corners (or in fact edges, cylinders or specular reflections). This is demonstrated in figure 16 where RCD's of angles greater $10^{\circ}$ and a range tolerance of less than $4 \mathrm{~cm}$ are shown, after extraction from a single scan. To form the 


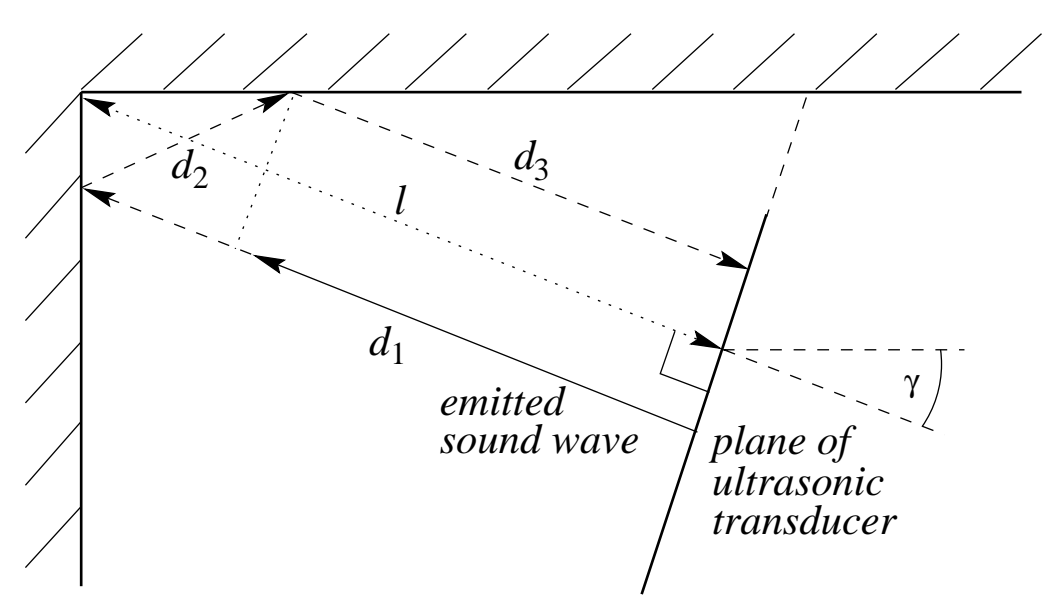

Figure 15: The geometry of ultrasonic reflection within the vicinity of a corner.

beginning of a simple navigation algorithm, Leonard proposed a tracking algorithm to monitor the relative motion of RCD's as a mobile robot moved through its environment [35]. He showed that hypotheses as to the origin of an RCD could be formed by firstly matching and then observing subsequent RCD's. In particular RCD's produced from a wall, translate tangentially along the wall, whereas RCD's produced from a corner rotate about the actual corner. These qualitative hypotheses are further demonstrated in figure 17 where RCD's extracted from 15 mobile robot positions are shown superimposed upon each other. Note that under the proposed model it is possible to extract most of the walls and corners.

By eventually finding the relative locations of walls and corners (and cylinders), Leonard and Durrant-Whyte demonstrated a simple mobile robot navigation technique with SONAR.

Another proposed method for the reliable recognition of discriminating features, is that of triangulation based fusion proposed by Wijk and Christensen [36]. The aim of this work was to use less signal processing than the above methods, and achieve less target localization accuracy, but still achieve reliable target recognition from a ring of SONAR transducers (Polaroid 6500 type) which are consecutively activated during vehicle motion. After each new scan is completed, a computational search is made for geometrical intersections between detected RCDs within the current and any previously stored scans. Again, once enough manoeuvres and scans had taken place, target classification and matching issues were addressed to aid vehicle localization. 


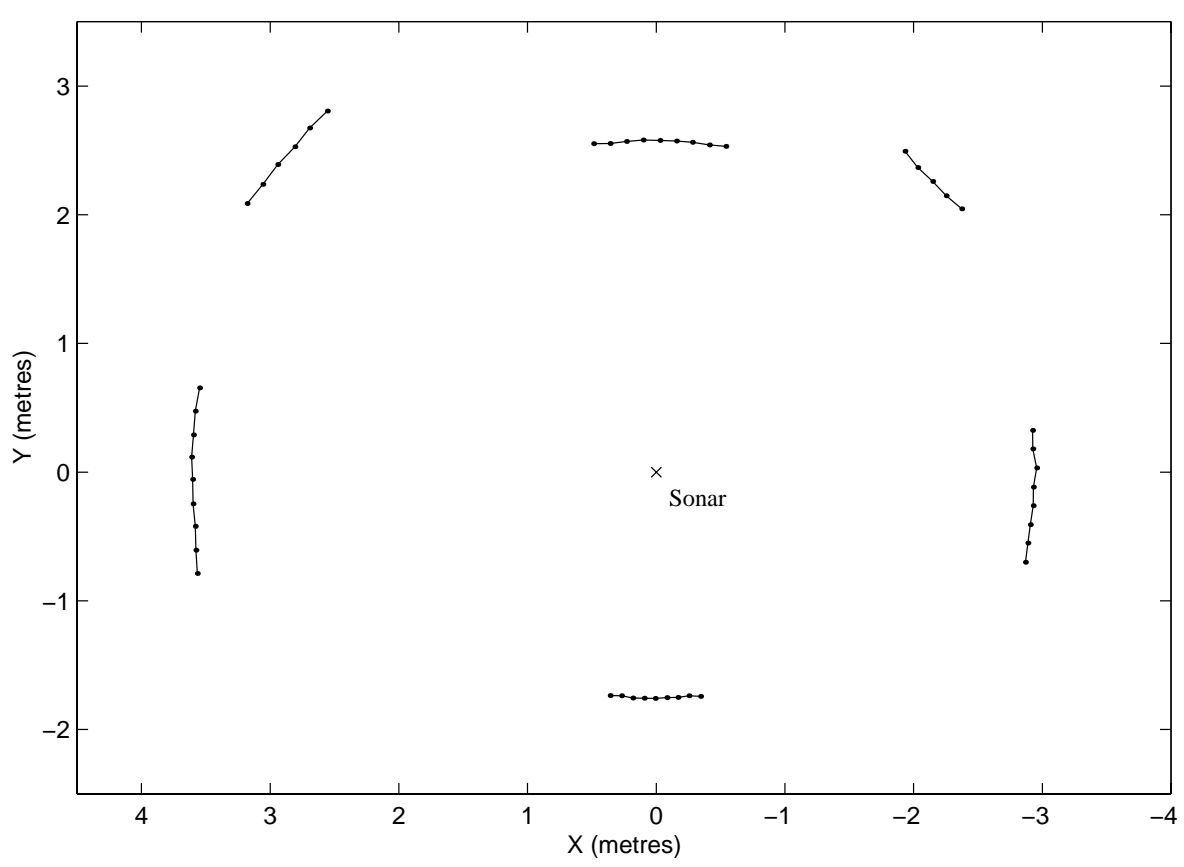

Figure 16: RCD's extracted from a single SONAR scan.

\subsection{Target Classification with SONAR Arrays}

More of the recent work on SONAR, relevant to robot navigation, attempts to achieve this definition of target recognition without the necessity of moving the SONAR(s).

Kleeman and Kuc published an article demonstrating the use of a SONAR array consisting of two ultrasonic transmitter/receiver transducers [37] (also referred to as double pulse coding [38]). They further accounted for the effects of temperature and humidity, producing a system which can reach an accuracy of $1 \mathrm{~mm}$ in range and $0.1^{\circ}$ in angular resolution in still air, while being able to discriminate between objects separated by $10 \mathrm{~mm}$, when measuring up to a maximum range of $8 \mathrm{~m}^{8}$.

In Kleeman's research, target classification, without sensor motion is carried out using a method known as template matching. Echo shapes for different transmitting and receiving angles and ranges are generated and stored. By then selecting the highest correlation match between the received echo and the template set, the optimal arrival times at the two transducers can be selected and hypotheses made as to the nature of the target.

\footnotetext{
${ }^{8}$ Other work which analyses the accuracy of $3 \mathrm{D}$ SONAR, applied to the problem of position estimation of a robot wrist was examined by Wehn and Belanger [39], in which a distinction was made between slowly changing atmospheric characteristics, which were modeled as "deterministic" and the high frequency aspects of the room atmosphere which were modeled as stochastic processes.
} 


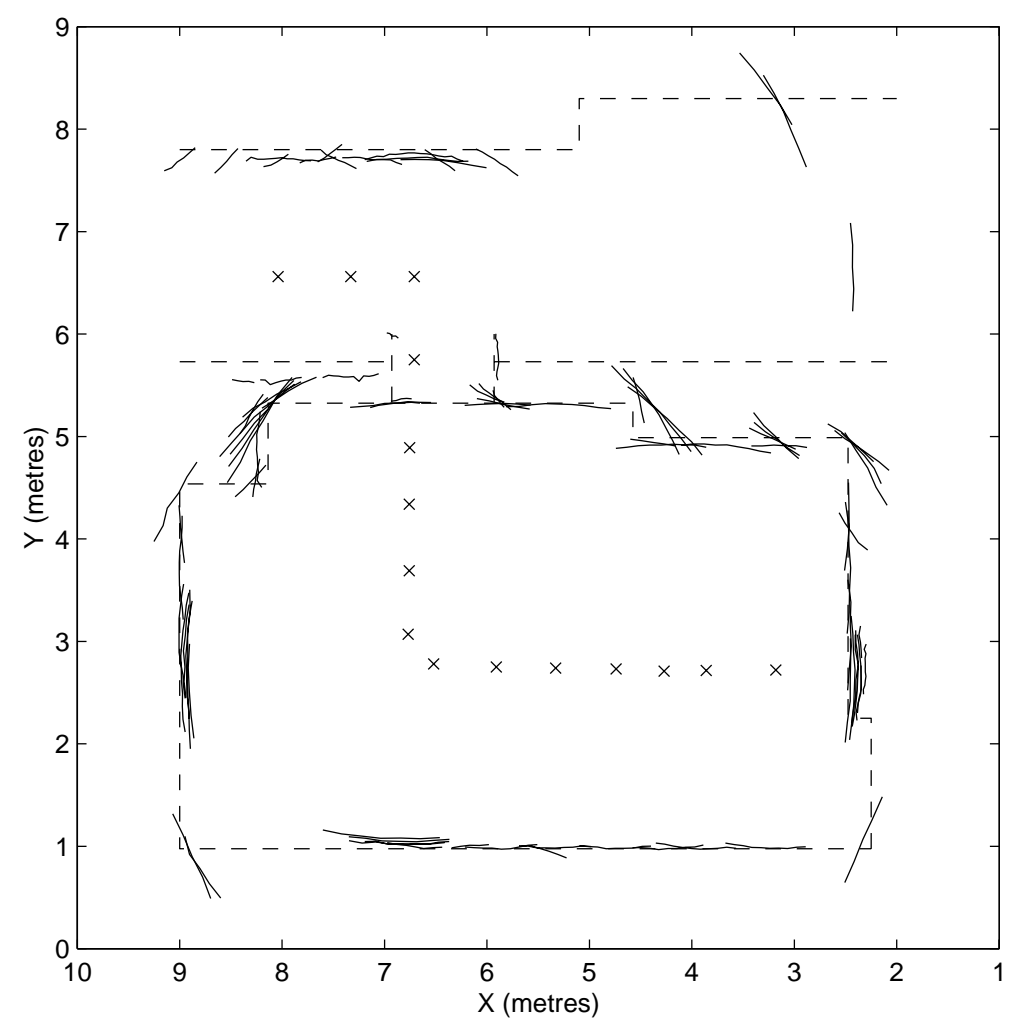

Figure 17: All RCD's extracted from 15 different sensor positions superimposed upon each other. The hand measured environment is represented by the dashed lines.

Recent work by Peremans et al. and Barshan et al. has further demonstrated the use of multisensor configurations - specifically using 3 Polaroid transducers to improve the location and radius of curvature estimation of objects in 2D [40,31]. The initial work of Kuc and Siegel demonstrated above shows that in order to recognize different types of reflectors, a series of sightings of the same object from different view points was necessary [30]. Peremans et al. demonstrate a novel method for immediate object recognition, based on radius of curvature, which utilizes a single transmit/receive ultrasonic transducer together with two extra receivers. It is shown that by estimating the precise arrival times of a single transmitted echo at each of the 3 receivers, the reflector type and once again, the location can be determined from a single view point. Barshan et al. further showed that when the reflection point of the object being sensed is not along the line of sight of the ultrasonic transducer, there is a decline in the amplitude of the reflected SONAR signal, which decreases the signal-to-noise ratio (SNR). They proposed a solution in which a minimum of 3 transmitting/receiving transducers are used. Depending on the location of an object, The sensor 
head can rotate each transducer individually about their centers, towards the target to obtain a higher SNR. This research focuses initially on improving TOF estimates using a curve fitting method, rather than just thresholding the returned acoustic signal. An adaptation process then takes place in which an initial estimate of the radius of curvature of an object is made with all 3 transducers in a flat configuration. After this the transducers are rotated and an improved estimate results. This process is repeated until the accuracy of the final curvature estimate reaches an acceptable level. The radius of curvature estimate provides useful information for differentiating different types of reflectors such as edges, cylinders and walls.

\subsection{Limitations of SONAR}

A consequence of the sensitivity of SONARs to retro-reflectors (as shown in figure 15) is that retro-reflectors lying outside of the scanning plane of the ultrasonic sensor can produce misleading range readings. Most of the research work in SONAR, applied to robot navigation, assumes planar models. For example, Kuc and Siegel stated that it is not possible for the floor to return a reflection at the point where the ultrasonic cone intersects it. This is true unless there is a discontinuity (eg. a small door step) which can return a very strong signal of the type demonstrated in figure 15. Hence, one danger of the wide beam width produced by SONAR sensors is that one cannot guarantee that the range data recorded actually corresponds to objects within the scanning plane of the sensor. Work which considers the $3 \mathrm{D}$ effects of transmitted ultrasonic waves can be found in [39].

Further limitations result due to the speed at which data can be extracted from SONAR. Due to the speed of sound in air, large distance measurement (greater than about $15 \mathrm{~m}$ ) becomes a slow process. If the settling time of the transmit/receive transducer is allowed for, new range information cannot generally be sampled at a rate of more than $3 \mathrm{~Hz}$ - i.e. the sensor head can only take new readings, from different orientations, 3 times a second, which is a very low data rate for autonomous vehicle applications. Also if a target is too far from a SONAR array, then the target classification methods in section 3.3 can fail. Once again this means that data from multiple view points is needed for target recognition and hence correct range-to-target estimation. This places a large time over head on any related robot navigation algorithm. 


\section{Summary}

In introducing different possibilities for recording range measurements in indoor environments, an attempt has been made to provide an unbiased view of the advantages and disadvantages of various coaxial methods used in mobile robotics.

It was shown that the correspondence and disparity problems associated with stereo vision can be eliminated with coaxial LIDAR sensors. These have a major advantage over triangulation systems, and in terms of processing the range data produced by these sensors, they offer a very simple and practical solution for range estimation in mobile robotics. Only in recent years have such sensors of reasonable size and cost become available for consideration in autonomous vehicle applications.

In this article various LIDAR sensors and their range detection methods have been reviewed. Real data was presented, from the SICK TOF LADAR and from AMCW LIDARS. The data has been analysed and various conclusions, regarding the applicability of each type of sensor, have been drawn with respect to sensitivity to environmental conditions (eg. temperature), optical/electronic cross-talk and pulse rise time fluctuations.

The concept of averaging range data for range estimate improvement was also reviewed and a method was presented which manipulates certain electronic and geometrical/optical limitations in any real LIDAR, to achieve this.

Without a doubt, the most famous sensor used within the mobile robotics community has been SONAR. The relatively low cost of many of the commercially available SONARs, along with their compact size, has made them a very attractive range measuring device. Although the data they produce does not directly appear to represent the environment, previous research has shown that, with a good physical model and understanding of SONAR reflection in air, these sensors can provide very useful information. It must also be said however, that environmental data acquisition using these sensors is a slow process. The source of the RCDs mentioned in section 3 for example, is ambiguous, and it is not until several scans from differing positions become available that useful information for navigation emerges. Indeed, in a sense the disparity problem shows itself with SONAR too, since it is possible for certain target configurations to reflect the radiated acoustic 
energy, such that the receiver is unable to detect it. A form of the correspondence problem also presents itself since the actual source of a particular reflection, and hence range reading, is not known precisely, but can only be narrowed down to a certain region within the beam width of the sensor.

To speed up the process of target classification, recent research has focussed on the use of SONAR arrays. Methods such as template matching have been reviewed which has been shown successful in allowing 3 SONAR transducers to estimate the type of target being sensed from a single location. The range to this target can then be estimated correctly.

Choosing a particular sensor, or combination of sensors, from the vast array of possibilities now available for indoor sensing, is not a trivial task. Indeed a full analysis of the qualities and short comings of all the different sensing possibilities in mobile robotics extends beyond the scope of this review. Indeed it can be noted from the literature, that a marked increase in the use of LIDAR has occurred in recent years, due to their reduced cost and the ease of obtaining reliable range data in comparison with SONAR, this often being the only affordable sensor 20 years ago.

Although interest in diverse sensing methods in robotics has increased in recent years, the full capability of sensors, in terms of low cost, compact design, optimal noise rejection, high speed data production and particularly optimal data processing, is very much in its infancy, and the sensing problem is therefore still a very extant research issue in robotics.

\section{Acknowledgments}

The author would like to thank Sarath Kodagoda (NTU) for contributing TOF SICK scans and Daniel von Fluee for his work with ultrasonic sensing, used in this article.

\section{References}

[1] D. Nitzan, A. E. Brain, and R. O. Duda. The measurement and use of registered reflectance data in scene analysis. In Proc. IEEE., pages 206-220, vol. 65, 1977.

[2] M. J. Brownlow. A Time of Flight Optical Range Sensor for Mobile Robot Navigation. PhD thesis, University of Oxford, United Kingdom., 1993.

[3] R. Jarvis. A laser time-of-flight range scanner for robotic vision. IEEE Trans. Pattern Analysis Machine Intell., 5:505-512, 1983 .

[4] O. Partaatmadja, B. Benhabib, A. Sun, and A.A. Goldenberg. An electrooptic orientation sensor for robotics. IEEE J. Robotics and Automation, 8(1), 1992. 
[5] A. Bonen, R.E. Saad, K.C. Smith, and B. Benhabib. A novel electroptical proximity sensor for robotics: Calibration and active sensing. IEEE J. Robotics and Automation, 13(3), 1997.

[6] T. Okada and U. Rembold. Proximity sensor using a spiral-shaped light emitting mechanism. IEEE J. Robotics and Automation, 7(6), 1991.

[7] P. W. Smith, N. Nandhakumar, and C. H. Chien. Object motion and structure recovery for robotic vision using scanning laser range sensors. IEEE J. Robotics and Automation, 13(1), 1997.

[8] D. C. Tseng and Z. Chen. Computing location and orientation of polyhedral surfaces using a laser-based vision system. IEEE J. Robotics and Automation, 7(6), 1991.

[9] M. Hebert and E. Krotkov. 3-D measurements from imaging laser radars: How good are they? In Int. Conf. Intelligent Robots and Systems, pages 359-364, 1991.

[10] Martin D. Adams. Sensor Modelling, Design and Data Processing for Autonomous Navigation. World Scientific, 1999.

[11] Inc. Schwartz Electro-Optics. Scanning laser rangefinder. Technical report, Product Literature, Schwartz Electro-Optics, Inc., 3404 N. Orange Blossom Trail, Orlando, FL 32804, U.S.A., 1991.

[12] Inc. Schwartz Electro-Optics. SHIELD Scanning Helicopter Interference Envelope Laser Detector. Technical report, Product Literature, Schwartz Electro-Optics, Inc., 3404 N. Orange Blossom Trail, Orlando, FL 32804, U.S.A., 1995.

[13] D. Pagac, E. M. Nebot, and H. F. Durrant-Whyte. An evidential approach to map-building for autonomous vehicles. IEEE J. Robotics and Automation, 14(4):623-629, 1988.

[14] W.S. Wijesoma, K.R.S. Kodagoda, A.P. Balasuriya, and E.K. Teoh. Road edge and lane boundary detection using laser and vision. In Int. Conf. Intelligent Robots and Systems, 2001.

[15] P. Vuylsteke, C.B. Price, and A. Oosterlinck. Image sensors for real-time 3D acquisition, part 1. In T.C. Henderson, editor, Traditional and Non-Traditional Robotic Sensors, pages 187-210. Springer Verlag, 1990.

[16] Inc. VORAD Safety Systems. The VORAD vehicle detection and driver alert system. Technical report, Product Literature, VORAD Safety Systems, Inc., 10802 Willow Ct, San Diego, CA 92127, U.S.A.

[17] B. Siuru. The smart vehicles are here. Popular Electronics, 1(1):41-45, 1994.

[18] G. L. Miller and E. R. Wagner. An Optical Rangefinder for Autonomous Robot Cart Navigation. Technical report, A.T\&T. Bell Laboratories, Princeton NJ., 1987.

[19] M. D. Adams. Optical Range Data Analysis for Stable Target Pursuit in Mobile Robotics. PhD thesis, University of Oxford, United Kingdom., 1992.

[20] M. D. Adams. Lidar design, use and calibration concepts for correct environmental detection. IEEE Transactions on Robotics and Automation, 16(6):753-761, 2001.

[21] J.A. Castellanos and J.D. Tardos. Mobile Robot Localization and Map Building: A Multisensor Fusion Approach. Kluwer Academic Publishers, Boston, 1st edition, 2000.

[22] W. D. Koenigsburg. Noncontact distance sensor technology. Technical Report 02254, GTE Laboratories, Inc., 40 Sylvan Rd., Waltham, MA, 1982.

[23] J. Borenstein and Y. Koren. Histogrammic in-motion mapping for mobile robot obstacle avoidance. IEEE J. Robotics and Automation, 7(4), 1991.

[24] J. L. Crowley. World modelling and position estimation for a mobile robot using ultrasonic ranging. In IEEE J. Robotics and Automation, pages 674-680, 1989.

[25] J. H. Ko, W. J. Kim, and M. J. Chung. A method of acoustic landmark extraction for mobile robot navigation. IEEE J. Robotics and Automation, 12(3), 1996.

[26] J. Leonard and H. F. Durrant-Whyte. Application of Multi-Target Tracking to Sonar Based Mobile Robot Navigation. In International Conference on Decision and Control, 1990.

[27] Polaroid Corporation Commercial Battery Division. Ultrasonic Ranging System. Polaroid Corporation, Cambridge, MA, U.S.A., 1984. 
[28] SNT Sensortechnik. Ultrasonic Displacement Sensors for Distances up to $5000 m m$. SNT Sensortechnik AG, CH8153 Ruemlang, Switzerland, 1994.

[29] Daniel von Fluee. Localisation und map building fuer mobile roboter. Technical report, Institute of Robotics, Swiss Federal Institute of Technology, Zurich, Switzerland, 1994.

[30] R. Kuc and M. W. Siegel. Physically Based Simulation Model for Acoustic Sensor Robot Navigation. In IEEE Trans. Pattern Analysis Machine Intell., pages 766-778, 1987.

[31] Ali Safak Sekmen and Billur Barshan. Estimation of object location and radius of curvature using ultrasonic sonar. Elsevier Science - Applied Acoustics, 62:841-865, 2001.

[32] B. Ayrulu and B. Barshan. Identification of target primitives with multiple decision-making sonars using evidential reasoning. Int. J. Robotics Research, 17(6):598-623, 1998.

[33] J. L. Crowley. Dynamic world modeling for an intelligent mobile robot using a rotating ultra-sonic ranging device. In Proc. IEEE Int. Conf. Robotics and Automation, page 128, 1985.

[34] S. Lang, L. Korba, and A. Wong. Characterizing and modelling a sonar ring. In SPIE Conference on Mobile Robots IV, pages 291-304, Philadelphia, U.S.A., 1989.

[35] John J. Leonard and Hugh F. Durrant-Whyte. Directed Sonar Sensing for Mobile Robot Navigation. Kluwer Academic Publishers, 1992.

[36] Olle Wijk and Henrik I. Christensen. Triangulation-based fusion of sonar data with application in robot pose tracking. IEEE Transactions on Robotics and Automation, 16(6):740-752, 2000.

[37] Lindsay Kleeman and Roman Kuc. Mobile robot sonar for target localization and classification. Int. J. Robotics Research, 14(4):295-318, 1995.

[38] L. Kleeman. Fast and accurate sonar trackers using double pulse coding. In Int. Conf. Intelligent Robots and Systems, pages 1185-1190, 1999.

[39] Hans W. Wehn and Pierre R. Belanger. Ultrasound-based robot position estimation. IEEE Transactions on Robotics and Automation, 13(5):682-692, 1997.

[40] Herbert Peremans, Koenraad Audenaert, and Jan M. Van Campenhout. A high resolution sensor based on tri-aural perception. IEEE Transactions on Robotics and Automation, 9(1):36-48, 1993. 